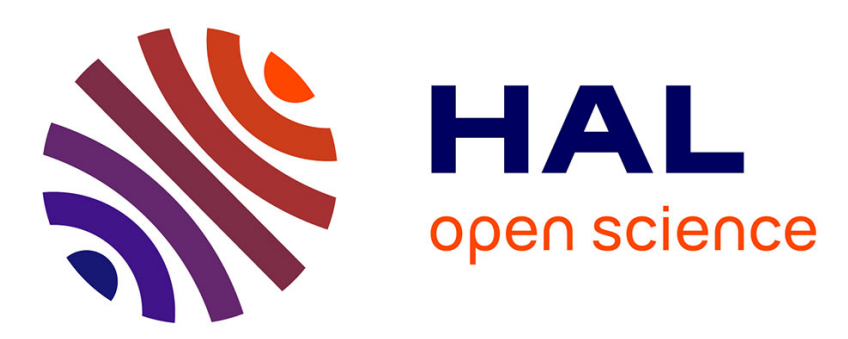

\title{
Les grandes tendances démographiques et l'environnement: l'enjeu d'une planète viable
}

Hervé Domenach

\section{To cite this version:}

Hervé Domenach. Les grandes tendances démographiques et l'environnement: l'enjeu d'une planète viable. Mondes en Développement, 2008, 36 (142), pp.97-111. ird-00430599

\section{HAL Id: ird-00430599 \\ https://hal.ird.fr/ird-00430599}

Submitted on 9 Nov 2009

HAL is a multi-disciplinary open access archive for the deposit and dissemination of scientific research documents, whether they are published or not. The documents may come from teaching and research institutions in France or abroad, or from public or private research centers.
L'archive ouverte pluridisciplinaire HAL, est destinée au dépôt et à la diffusion de documents scientifiques de niveau recherche, publiés ou non, émanant des établissements d'enseignement et de recherche français ou étrangers, des laboratoires publics ou privés. 


\title{
Les grandes tendances démographiques et l'environnement : l'enjeu d'une planète viable
}

\section{Hervé DOMENACH ${ }^{1}$}

\begin{abstract}
T a régulation démo-environnementale planétaire concerne tout à la fois les 1 territoires, les nations et les individus. Mais dans les conditions politiques et institutionnelles actuelles, cette (dé)régulation ne trouve le plus souvent que des solutions partielles au niveau local ou régional, ce qui pose en préalable la question du développement global et donc de la solidarité internationale et de ses règles. C'est en 1951 que fut publié le premier rapport sur l'environnement dans le Monde par l'UICN (Union Internationale pour la Conservation de la Nature), mais il fallut attendre la fin des années 1960 pour qu'émerge une relative prise de conscience des nuisances de la croissance économique: déchets, pollutions de l'air, de l'eau et des sols, tandis que le questionnement concernant la surexploitation des écosystèmes et l'épuisement des ressources naturelles fut pris en compte pour la première fois en 1972 dans le rapport The limits to growth publié par le Club de Rome.
\end{abstract}

En 2008, les défis environnementaux sont devenus très complexes, concernant essentiellement le changement climatique, l'appauvrissement de la biodiversité, la gestion non durable des sols et des ressources en eau, les conséquences sanitaires de la pollution et des produits chimiques dangereux..., posant ainsi la question nécessairement politique d'une gestion viable à terme de notre environnement planétaire.

Parallèlement, de grandes tendances démographiques se sont affirmées au tournant de ce siècle : les baisses de la fécondité et de la croissance naturelle sont confirmées et l'on sait que le croît démographique restera élevé dans de nombreux pays pendant trois décennies environ, en raison des effectifs encore importants de population en âge de reproduction. Au regard de ces évolutions, la recherche s'est logiquement tournée vers les questions interdisciplinaires d'accompagnement de cette croissance démographique, souvent marquée par un héritage de pauvreté, d'analphabétisme, de sous-équipement, de carences relatives d'alimentation, d'hygiène et de santé. Se pose, en conséquence, la question des politiques de développement qui doivent assurer à tous les individus une amélioration substantielle de leurs conditions de vie, une garantie

1 Démographe, Directeur de recherches IRD-Laboratoire Population-EnvironnementDéveloppement. domenachhh@wanadoo.fr 
d'accès aux ressources essentielles et un développement humain et social de qualité. Si l'on suit ce raisonnement, la valorisation de l'environnement sous toutes ses formes n'est alors qu'une résultante parmi d'autres des améliorations sociétales: éducation, santé de la reproduction, aménagement du territoire, gestion des ressources.

Or, il faut aborder également la relation population-environnement à partir des dégradations et des nuisances observées à différentes échelles spatiales, ce qui amène à l'observation suivie des paramètres de base: l'air, l'eau, les sols, les forêts, qui sous-tendent les études portant sur les enjeux environnementaux: effet de serre et changements climatiques, gestion des déchets, énergies renouvelables, gestion des milieux naturels à long terme, etc. Ces enjeux se conjuguent dans la question du développement durable en tant que solidarité diachronique entre les générations, dans la mesure où la garantie d'accès aux ressources doit être assurée pour les générations futures, ce qui conduit à considérer également les différentes échelles de temps.

Ainsi, la croissance démographique n'est que l'une des causes de l'exploitation accrue des ressources disponibles et de la gestion souvent incontrôlée de l'espace et n'est pas nécessairement le facteur le plus déterminant de la dégradation de l'environnement. La transformation des besoins joue également un rôle majeur, de même que les mutations familiales et sociales, les logiques de production de rente associée à l'absence de sécurité de la tenure foncière, le désenclavement des campagnes et l'urbanisation.

En caricaturant, on peut considérer que deux tendances théoriques s'affrontent, renvoyant à deux analyses idéologiques différentes. Pour l'une, la croissance démographique est la source de tous les drames humains : guerres, épidémies, famines, pauvreté... et aujourd'hui dégradation de l'environnement. Pour l'autre, elle n'est qu'un facteur spécifique dont le rôle n'est pas nécessairement déterminant et ne comporte pas que des aspects négatifs, l'aménagement concerté des territoires, l'agglomération et la densification de la population pouvant devenir des vecteurs du développement et d'une meilleure gestion des milieux. Des néo-malthusiens convaincus aux anti-malthusiens non moins convaincus, la gamme des opinions s'est aujourd'hui considérablement élargie, en raison des connaissances acquises en écologie et sur l'évolution des populations. Pour autant, la question de la synthèse analytique de la relation population-environnement reste entière et la sophistication des corrélations de variables issues de l'observation doit impérativement être évaluée à l'aune des choix politiques et des échelles décisionnaires.

La population de neuf milliards attendue pour 2040, selon les perspectives établies à ce jour, connaitra des changements de structure très importants et un certain nivellement des différences entre les continents. Sera-t-elle constituée d'une majorité de personnes âgées comme le prévoient les projections, sera-telle entassée dans de grandes métropoles, disséminée dans les campagnes et les territoires encore peu occupés, connaitra-t-elle de grandes migrations occasionnant de nouvelles répartitions territoriales? Parmi d'autres moins prégnantes, ces trois questions sur le vieillissement, l'urbanisation et la mobilité 
conditionneront la nature des relations des hommes avec les facteurs fondamentaux de leur environnement: l'eau, l'air, les sols, la sécurité alimentaire.

\section{LE CROÎT DÉMOGRAPHIQUE ET LES CONSÉQUENCES DU PROCESSUS DE VIEILLISSEMENT}

Selon un compteur symbolique de la population mondiale ${ }^{2}$, la terre comptait 6683387000 individus au 11 mars 2008. On estime ainsi que la planète enregistre chaque jour environ 350000 naissances et 160000 décès, soit 190000 bouches supplémentaires à nourrir. Mais la lente progression de l'espèce humaine n'a eu d'effets majeurs sur l'environnement que très récemment et le sentiment que la terre est prodigue de ses ressources reste encore bien ancré dans la mémoire collective, entravant la prise de conscience des déséquilibres démo-environnementaux présents et à venir.

Malgré la baisse de fécondité enregistrée dans la plupart des pays, avec un net retard dans le continent africain, il faudra attendre encore trois à quatre décennies pour que la population mondiale connaisse un renouvellement équilibré, en raison du poids des générations issues des périodes antérieures de forte fécondité. Sachant que 95\% de l'accroissement démographique en cours dans le monde concerne les pays du Sud et que leur importance dans la population mondiale passerait de $68 \%$ en 1950 à plus de $85 \%$ vers les années 2050 selon les estimations de la Division de la Population des Nations Unies ${ }^{3}$, on risque d'assister à une formidable redistribution démographique entre les différents continents et pays : en 2050, l'Inde deviendrait le pays le plus peuplé (1 530 millions d'habitants), devançant la Chine (1 400 millions) et de loin les États-Unis (408 millions) puis le Pakistan (348 millions), tandis que l'Afrique compterait trois fois plus d'habitants que l'Europe, alors que la situation était exactement inverse en $1950^{4}$. En revanche, quelques pays, occidentaux surtout, risquent d'affronter des phénomènes de dépopulation très préoccupants : entre autres nombreux exemples, la Russie passerait de 145 millions en 2000 à 101 millions en 2050, si elle maintenait sa fécondité de 1,2 enfants par femme!

La forte croissance des pays du Sud a rajeuni la population mondiale, et pourtant se profilent déjà les signes d'un processus de vieillissement qui atteindra probablement la quasi-totalité des pays à terme. La baisse de la

2 Cf. www.worldpopclock.com. Nombre de pays ne disposant pas d'un système censitaire fiable, ou ayant des données trop anciennes, on peut considérer que l'incertitude concernant les effectifs de population mondiale est approximativement de 2 à $3 \%$.

${ }^{3}$ Les projections de population dépendent des hypothèses formulées pour la fécondité, la mortalité, les migrations, et nécessitent donc des réajustements permanents. Hors évènements de rupture (guerre, épidémies, cataclysmes...), les évolutions démographiques sont cependant suffisamment lentes pour offrir une relative fiabilité à moyen terme.

${ }^{4}$ En 2050, l'Afrique atteindrait 1969 millions d'habitants et l'Europe 660 millions, contre respectivement 224 millions et 549 millions un siècle auparavant (1950) (World data sheet, population reference Bureau, 2005).

Mondes en Développement Vol. 36-2008/2-n¹42 
fécondité et la plus grande longévité des individus sont à considérer comme des tendances lourdes des évolutions futures des populations, elles tendent à diminuer la part des enfants et à tirer la structure par âge vers les âges élevés : l'âge médian de la population mondiale passera ainsi probablement de 26 ans actuellement à près de 50 ans vers 2050 .

En 2005, 28,3\% des Terriens ont moins de 15 ans, contre 10,3\% âgés de 60 ans et plus, tandis qu'en 2050 la proportion des "moins de 15 ans" ne serait plus que de 19,8\% et celle des "60 ans et plus" doublerait, atteignant 21,8\% (tableau 1). Cette évolution spectaculaire répond à deux facteurs : la baisse de la fécondité et l'espérance de vie. La première, partout dans le monde, décroît : 2,7 enfants par femme en moyenne aujourd'hui, qui tendrait vers 2,1 enfants en 2050, soit précisément le seuil de renouvellement démographique. Cette perspective peut sembler étonnante si l'on considère que les femmes maliennes et nigériennes font encore plus de 7 enfants en moyenne ${ }^{5}$ aujourd'hui, mais il s'agit de situations extrêmes qui n'intègrent pas encore les éléments d'une transition démographique entamée, que l'on observe par ailleurs. Quant à l'espérance de vie à la naissance, en moyenne de 65 ans aujourd'hui, elle augmente continuellement et serait au-delà de 70 ans en 2050, mais la dispersion reste considérable : 82 ans au Japon contre seulement 37 ans en Zambie!

Tableau 1 : Distribution de la population mondiale (en \%), par grands groupes d'âge et aire géographique, en 2005 et projection en 2050 (hypothèse moyenne)

\begin{tabular}{|l|c|c|c|c|c|c|c|c|}
\hline & \multicolumn{4}{|c|}{2005} & \multicolumn{4}{c|}{2050} \\
\hline & $0-14$ & $15-59$ & + de 60 & + de 80 & $0-14$ & $15-59$ & + de 60 & + de 80 \\
\hline Total Monde & 28,3 & 61,4 & 10,3 & 1,3 & 19,8 & 58,3 & 21,8 & 4,4 \\
\hline Régions plus développées & 17,0 & 62,9 & 20,1 & 3,7 & 15,2 & 52,2 & 32,6 & 9,4 \\
\hline Régions moins développées & 30.9 & 61.0 & 8.1 & 0.8 & 20.6 & 59.3 & 20.1 & 3,6 \\
\hline Afrique & 41,4 & 53,4 & 5,2 & 0,4 & 28,0 & 61,7 & 10,4 & 1,1 \\
\hline Asie & 28.0 & 62.7 & 9.2 & 1.0 & 18.0 & 58.3 & 23.7 & 4.5 \\
\hline Europe & 15.9 & 63.5 & 20.6 & 3.5 & 14.6 & 50.9 & 34.5 & 9.6 \\
\hline Amérique latine et Caraibes & 29.8 & 61.2 & 9.0 & 1.2 & 18.0 & 57.8 & 24.3 & 5.2 \\
\hline Amérique du Nord & 20.5 & 62.7 & 16.7 & 3.5 & 17.1 & 55.6 & 27.3 & 7.8 \\
\hline Océanie & 24,9 & 61,0 & 14,1 & 2,6 & 18,4 & 56,9 & 24,8 & 6,8 \\
\hline
\end{tabular}

Source : World population prospects, the 2006 revision, Highlights, FNUAP, ONU, New York.

Cinq ans après la deuxième Assemblée mondiale de l'ONU sur le vieillissement, qui s'était déroulée à Madrid en 2002, les ministres des États d'Europe et d'Amérique du Nord, ainsi que de plusieurs États d'Asie centrale se sont rencontrés en Espagne en 2007 avec pour mot d'ordre de «favoriser le développement d'une société pour tous les âges ». L'augmentation du nombre des personnes âgées est une conséquence de la baisse des taux de fécondité et de mortalité combinée à l'augmentation considérable de l'espérance de vie, et le vieillissement de la population est sans doute le phénomène le plus préoccupant auquel les sociétés humaines devront faire face dans les prochaines décennies car il concernera tous les pays, hors hypothèse de rupture économique,

${ }^{5}$ Cf. Tous les pays du monde, Population et sociétés n 414, juillet 2005.

Mondes en Développement Vol.36-2008/2-n¹42 
épidémiologique ou environnementale. Les perspectives démographiques sont claires sur ce point : à l'échelle mondiale, le nombre de personnes âgées de 60 ans et plus, devrait passer de 673 millions en 2005 à quelques 2 milliards vers 2050, tandis que le nombre de personnes âgées de 80 ans et plus, passerait de 88 millions en 2005 à 400 millions environ vers 2050 et que le nombre des centenaires pourrait atteindre alors 3 à 4 millions d'individus.

C'est dans les actuels pays dits du Sud que le vieillissement s'opérera le plus rapidement, puisque les projections indiquent que $80 \%$ des plus de 60 ans vivront alors dans ces pays qui verront la proportion de leurs seniors passer en moins d'un demi-siècle de 8 à $20 \%$ de leur population. À titre d'exemple, s'il a fallu 115 ans, pour que la proportion de personnes âgées double en France, il ne faudra que 27 ans pour que le même phénomène se produise en Chine, ce qui a fait dire à Mme Brundtland, directrice générale de l'Organisation mondiale de la santé, que "tandis que les pays développés sont devenus riches avant de devenir vieux, les pays en développement seront vieux avant de devenir riches". Si l'on considère que le vieillissement a des répercussions majeures sur tous les aspects de la vie humaine : la croissance économique, l'épargne, l'investissement et la consommation, le marché du travail et les retraites, la fiscalité et les transferts intergénérationnels, la santé et les soins médicaux, la composition des familles, les conditions de vie, le logement et les migrations, le passage d'un monde plutôt jeune à un monde plutôt vieux, impliquera, par contrecoup, de profondes mutations des sociétés et il faut s'attendre à des répercussions majeures sur nos modes vie qui détermineront des usages nouveaux de nos ressources énergétiques, aquatiques, alimentaires, etc.

\section{L'ENVIRONNEMENT ET LA MOBILITÉ DES HOMMES}

La révolution des technologies et des moyens de communication a provoqué une accélération de la mobilité humaine et une forte relativisation des distances, tandis que la révolution des transports par l'aménagement des réseaux terrestres et des espaces aériens a considérablement favorisé la croissance économique. Ainsi, la consommation d'espace va croissante et conditionne la recomposition des territoires et de leurs usages. Elle a aussi été la source de multiples nuisances et atteintes à l'environnement: consommation énergétique élevée, pollution atmosphérique, asphaltisation, dégradation des écosystèmes et des paysages. La priorité donnée aux transports routiers dans les pays occidentaux, qui pourtant bénéficiaient d'infrastructures fluviales et ferroviaires, a eu d'importantes répercussions sur l'environnement mais surtout elle a conduit les pays émergents à faire de même, et donc à négliger les infrastructures moins polluantes. Ainsi, dans les pays du Sud, les programmes d'investissement pour développer les réseaux ferroviaires ou fluviaux sont négligeables en rapport des fonds investis dans les réseaux routiers. On conçoit aisément l'incroyable facteur de pollution que déterminerait une densité de transport automobile 
dans les pays du Sud égale à celle que connaissent aujourd'hui l'Amérique du Nord, les pays européens et déjà certains pays émergents. Cet aspect global de la dynamique démographique semble pourtant largement irréversible, car il s'accorde avec un modèle social de plus en plus individualisé.

Plus largement, les activités humaines ont augmenté et entraîné le déplacement forcé de millions de personnes, soit pour cause de situations catastrophiques (conflits armés, accidents industriels, pollutions chimiques), soit qu'il s'agisse d'infrastructures (barrages, exploitations minières...), soit encore que cela résulte de processus de dégradation des écosystèmes terrestres, fluviaux et marins (concentrations foncières, épuisement des sols, salinisation...). Sont ainsi apparus, au début des années 1980, les premiers réfugiés environnementaux, en référence aux personnes déplacées pour cause de déforestation, réchauffement climatique, désertification, catastrophes naturelles et industrielles. Mais la convention de Genève (1951) ne couvre pas les réfugiés de l'environnement, considérant qu'un réfugié est une personne qui souffre de persécution "pour des raisons de race, de religion, de nationalité, de son appartenance à un groupe social particulier ou de ses opinions politiques ». Essam El-Hinnawi (1985) propose des critères de définition des réfugiés environnementaux dans un rapport du Programme des Nations Unies pour l'Environnement (PNUE), ouvrant la voie à un processus de catégorisation selon des typologies effectuées à partir du caractère temporaire ou non de la migration, la possibilité ou non de retourner sur les lieux de vie, tandis que d'autres définitions se référent par ailleurs à des réfugiés au sens de critères plus strictement écologiques. Dans un avenir peut-être proche, le nombre de réfugiés de l'environnement pourrait augmenter de façon spectaculaire en raison de facteurs climatiques menant à l'érosion des sols, du réchauffement planétaire avec ses conséquences sur les territoires et les océans, de la pollution de l'eau, etc. Ce qui a conduit le Groupe d'experts intergouvernemental sur l'évolution du climat (GIEC), organe scientifique chargé par les Nations Unies d'examiner les causes et les effets des changements climatiques, à envisager 200 millions de réfugiés écologiques d'ici 2050. Citons quelques exemples: en Chine, selon la Banque asiatique de développement, près de 4000 villages sont menacés par la désertification et le désert de Gobi s'étend de 10400 kilomètres carrés supplémentaires chaque année. Et si, par exemple, la température moyenne de la planète augmentait, ne serait-ce que d'un degré ou deux, dans les décennies à venir ? La question est désormais posée institutionnellement et il s'agit d'en anticiper les multiples conséquences, et particulièrement ce qu'il adviendrait des populations concernées par la montée des océans (Bangladesh, littoral chinois, îles... mais aussi Pays-Bas et Floride), la disparition de terres arables, la désertification et la diminution de la fertilité des sols (Afrique, Moyen-Orient, Chine, Inde, Pakistan, mais aussi Espagne, Grèce, Portugal, soit une centaine de pays dans le monde), les famines liées aux sécheresses, les épidémies, etc. Il est probable que la planète n'en soit encore qu'aux prémices d'un processus intense de mobilité spatiale résultant des mutations environnementales. 


\section{LE PROCESSUS DE CONCENTRATION DES POPULATIONS ET LES MUTATIONS DÉMO-SPATIALES}

L'année 2007 marque un moment démographique essentiel : pour la première fois, parmi les 6,6 milliards de Terriens, il y a autant d'urbains que de ruraux selon les Nations Unies (2007a), alors que les urbains ne représentaient que 2\% en $1800,10 \%$ en 1900 , et seront probablement $75 \%$ en 2050 . Cependant, les urbains et les ruraux ne sont pas toujours clairement dissociables puisque dépendant de définitions non homogènes entre nations, de l'incertitude des comptages, notamment dans les agglomérations paupérisées, et de critères de densité et d'espacement de l'habitat qui n'intègrent pas la mobilité humaine croissante. Le rapport des Nations Unies confirme néanmoins le processus irréversible d'urbanisation, avec une estimation d'un million de citadins supplémentaires chaque semaine, en raison à la fois du croît démographique urbain endogène et de l'attraction de ruraux à la recherche d'un travail, d'un logement, de soins et d'éducation. Cette croissance rapide concerne principalement les agglomérations de 500000 habitants en moyenne qui abritent $52 \%$ de la population urbaine mondiale, tandis que la population des mégapoles ${ }^{6}$, qui rassemble aujourd'hui $4 \%$ de la population mondiale totale et $9 \%$ de la population urbaine totale, devrait suivre une croissance lente ${ }^{7}$, estimée à $3 \%$ par an environ. Mais cela se traduit souvent par un développement urbain anarchique et des conditions humaines, sanitaires et écologiques précaires. Vers 2030, la population urbaine pourrait dépasser les 5 milliards et tendrait vers plus de six milliards en 2050, et, parallèlement, le rapport estime la population actuelle des "taudis" du monde à un milliard, effectif impossible à projeter. On ne compterait alors plus que 39\% de ruraux dans le monde selon les projections des taux d'urbanisation ${ }^{8}$, alors que dans le même temps, la croissance de la population urbaine du monde développé passerait seulement de 870 millions d'habitants à 1,02 milliard. Comment héberger en 2050 trois à cinq milliards de citadins supplémentaires? Pourrons-nous assurer l'équilibre de l'écosystème planétaire sans limiter notre croissance économique ni modifier nos modes de production et de consommation?

La concentration des hommes en milieux urbains et l'accroissement des activités qu'ils y déploient interrogent l'avenir et la responsabilité des sociétés concernant leur mode de consommation des ressources et le traitement de leurs déchets. Si les pays industrialisés sont parvenus à relativement maîtriser les principales fonctions urbaines telles que l'habitat, les infrastructures et les équipements, les réseaux de transports, il en va différemment pour les autres pays où modernité et pauvreté coexistent souvent et qui sont largement

${ }^{6}$ Quoiqu'il y ait plusieurs définitions possibles pour qualifier une mégapole, on considère généralement qu'il s'agit de concentrations de population de 8 millions d'habitants et plus, avec une continuité de l'habitat au sens de l'agglomération.

7 Tokyo est aujourd'hui l'agglomération la plus importante avec 33 millions d'habitants.

${ }^{8}$ Le taux d'urbanisation mesure le nombre de citadins pour cent habitants.

Mondes en Développement Vol. 36-2008/2-n¹42 
confrontés à des règlementations partielles ou inadaptées, des infrastructures médiocres ou inexistantes dans les secteurs vitaux tels que la voirie, l'adduction d'eau potable, l'alimentation en électricité, les réseaux d'assainissement. À l'inverse, les concentrations humaines facilitent l'administration logistique, réduisent les coûts de gestion des transports, des services sanitaires, de sécurité, d'éducation... et limitent les déperditions de productivité. D'aucuns considèrent ainsi que l'urbanisation contient les remèdes à ses propres déséquilibres, en permettant une meilleure préservation des ressources naturelles et une meilleure gestion des effectifs de population à investissement égal. De fait, la concentration urbaine n'entraîne pas automatiquement la pollution, la dégradation des ressources, la surproduction de déchets. Ceux-ci sont dus principalement à des modes de production et de consommation non durables et aux carences d'une bonne gestion urbaine. Concentrant la moitié de la population du globe sur moins de $3 \%$ de la surface émergée, les villes sont ainsi susceptibles d'offrir de bonnes perspectives de durabilité à long terme.

Rares sont les organes décisionnels qui prennent en compte le fait que les mutations environnementales dépendent beaucoup des modes de consommation des populations urbaines et des filières agricoles productrices et qu'il importe donc de privilégier une vision globale du territoire et de mesurer les conséquences de décisions sectorielles dont la rentabilité économique à court terme constitue souvent l'unique critère. Entre de multiples exemples de ces situations, le rapport des Nations Unies cite les forêts tropicales de Tabasco (Mexique) qui ont été rasées afin de créer des zones d'élevage bovin pour satisfaire l'accroissement de la demande de viande de la ville de Mexico, distante de 400 kilomètres. Les politiques étatiques qui favorisent l'implantation de grandes exploitations agro-commerciales au détriment des petits producteurs créent des situations de rupture des écosystèmes qui entraînent souvent indirectement des processus de désertification, de déforestation ou d'érosion, etc. Se pose ainsi le problème de la répartition spatiale des populations à terme : les projections moyennes pour 2050 indiquent que 56 millions d'hectares seraient nécessaires pour accueillir les activités économiques non agricoles et pour loger les 1,5 milliards de nouveaux habitants dans les villes existantes, auxquels il faudrait ajouter 23 millions d'hectares pour les besoins en habitat des 2,5 milliards environ de nouveaux habitants attendus dans les villes à croissance informelle. Il est probable que la planète n'en soit encore qu'aux prémices d'un processus intense de mobilité spatiale résultant de la croissance urbaine et des mutations environnementales et il s'agit désormais d'en anticiper les multiples conséquences. Dans un contexte planétaire où la richesse des uns, tout autant que l'extrême pauvreté des autres, constituent de graves menaces pour l'environnement, n'apparaissent toujours pas clairement les politiques à mener pour surmonter les antagonismes entre le monde rural et le monde urbain, entre la satisfaction des besoins primaires et la surconsommation, entre les acteurs des pollutions et ceux qui les subissent.

Ainsi, la recomposition des espaces ruraux et les mutations des écosystèmes résultent à la fois de la distribution spatiale des populations et des pressions

Mondes en Développement Vol.36-2008/2-n¹42 
agro-industrielles; en contrepoint, les populations urbaines tendent à former de nouveaux socio-systèmes largement déconnectés de leur environnement physique et sollicitent toujours plus de ressources faisant appel à des logiques marchandes globalisées. L'agriculture structure de moins en moins les espaces ruraux et cette involution se traduit par un bouleversement de notre relation à la biosphère. La révolution agricole contemporaine a multiplié plusieurs dizaines de fois la productivité agro-industrielle, le rapport de productivité entre l'agriculture manuelle et l'agriculture moto-mécanisée moderne étant au tournant du siècle, de l'ordre de 1 à $500^{9}$ (Hamelin, 2004). On estime que les sols cultivables représentent $22 \%$ des terres émergées, soit 3300 millions d'hectares environ dont 1.500 millions d'entre eux sont cultivés aujourd'hui, tandis que les autres 1800 millions hectares potentiellement cultivables, dont 1400 dans les pays du sud, sont soumis à des fortes contraintes. Pour autant, les phénomènes démographiques sont finalement loin d'être des éléments fondamentaux au regard du creusement des inégalités et de la partition du monde entre populations nanties et les autres : un sixième de la population mondiale reçoit $78 \%$ des revenus mondiaux et contribue à $80 \%$ à la pollution de la planète; les tendances récentes à la libéralisation du commerce, à la déréglementation des marchés et à la mondialisation des capitaux, conduisent à une fracture toujours plus grande des écosystèmes traditionnels et provoquent la précarité des populations agricoles. Or, l'urgence est aujourd'hui de permettre aux pays les plus pauvres d'accueillir les 2,5 milliards d'hommes supplémentaires qui s'y concentreront dans les prochaines décennies.

\section{LA PRESSION DÉMOGRAPHIQUE ET LES RESSOURCES AQUATIQUES ET FORESTIÈRES}

Ce début du troisième millénaire voit se poser avec acuité la question de la limitation des ressources en eau, des régions arides, et des sécheresses récurrentes, la rareté des ressources conditionnant le développement socioéconomique. De la même manière, le rôle fondamental des forêts est de plus en plus fragilisé, ce qui affecte la conservation de la biodiversité et des sols, les cycles hydrologiques et les nappes phréatiques, ainsi que le stockage du dioxyde de carbone dans le contexte du changement climatique, sans oublier que les écosystèmes forestiers assurent la survie de millions d'hommes. Il fallut attendre les années 1970 (conférence de Mar del Plata, 1977) pour que la prise de conscience d'un risque de pénurie en eau conduise à des stratégies de gestion intégrée des ressources naturelles, cadrées par les diverses conventions internationales (Agenda 21 de la CNUED ${ }^{10}$ à Rio en 1992; Convention internationale contre la désertification, Paris, 1994 ; Conférence mondiale sur

\footnotetext{
${ }^{9}$ Pour produire la même valeur ajoutée qu'un travailleur agricole Danois il faut 1,3 Français ou Etasuniens, 2,2 Espagnols, 11 Brésiliens, 40 Égyptiens, 210 Bengalis, 610 Mozambicains.

${ }^{10}$ Conférence des Nations Unies sur l’Environnement et le Développement.
} 
l'eau, Paris, 1998 ; World water forum, Kyoto, 2003 ; Programme mondial pour l'évaluation des ressources en eau, Saragosse, 2004 ; $4^{\text {ème }}$ forum mondial de l'eau, Mexico, 2006, etc.).

Le stock d'eau de la planète est estimé à 1340 millions de milliards de $\mathrm{m}^{3}$. Mais l'eau douce n'en constitue qu'un peu moins de 3\% (soit 40 millions de milliards de $\mathrm{m}^{3}$ ), dont seulement $0,26 \%$ sont facilement exploitables pour les trois secteurs d'usages humains : l'agriculture qui représente $69 \%$ des prélèvements mondiaux, l'industrie $21 \%$ - dont plus de $80 \%$ sont restitués en eaux usées -, et la consommation humaine $9 \%$. Le Moyen-Orient, une majorité de pays africains et quelques autres pays, parmi lesquels l'Inde, la Belgique, la Pologne, seront bientôt confrontés à des besoins en eau qu'ils pourront difficilement satisfaire. La répartition des ressources par continent masque de fortes disparités régionales dans les grands pays comme le Brésil ou la Chine qui restent globalement bien pourvus en eau douce, alors que de vastes régions de leur territoire connaissent des problèmes d'aridité, invalidant toute perspective de développement économique et donc de maintien des populations. À l'inverse, certains pays traversés par des fleuves importants peuvent paraitre à l'abri des pénuries d'eau mais sont totalement dépendants des pays riverains pour la réelle disponibilité de cette eau pour les populations, tandis que d'autres doivent faire face à des dégradations : entre 1997 et 2000, les inondations ont recouvert pendant des mois les deux tiers du Bangladesh, impacté des millions de foyers chinois, ravagé une partie de l'Amérique centrale... Les perspectives mondiales sont préoccupantes, d'autant que la demande va s'accroitre considérablement et alourdir les difficultés en matière de sécurité d'approvisionnement et de santé. Le Sommet de la Terre à Johannesburg, en 2002, avait affirmé une ambition: faire qu'en 2015 le nombre des personnes dépourvues d'accès à l'eau potable ${ }^{11}$ soit diminué de moitié, ce qui suppose, outre la régulation démographique annoncée, de maitriser l'urbanisation anarchique, les pollutions liées à l'agriculture et à l'industrie, les conflits entre nations, etc. On mesure ainsi toute l'importance de ces rencontres internationales thématiques parce que même si les délais annoncés ne sont pas tenus, se met progressivement en place une gouvernance planétaire du bien public environnemental.

Il en va de même concernant les enjeux internationaux forestiers (biodiversité, changement climatique, désertification...) et la lutte contre les effets de serre : lors des récents sommets internationaux sur l'effet de serre (Kyoto, 1997 ; La Haye, 2000 ; Bali 2006, etc.), plusieurs pays, dont les États-Unis principalement, ont œuvré pour la mise en place au niveau international d'un marché des permis d'émission de gaz à effet de serre. Le principe d'une telle régulation apporte une nouvelle dimension potentielle de la mondialisation des enjeux forestiers, l'intérêt économique de ces activités forestières dépasserait alors largement la simple exploitation des bois. Mais par ce mécanisme, des acteurs financiers puissants pourraient acquérir des droits sur des territoires forestiers

${ }^{11}$ Population estimée en 2008 à 1,2 milliard par le Conseil mondial de l'eau. 
situés dans des pays défavorisés, ce qui conduirait à une prise de contrôle de l'espace et des ressources au détriment des acteurs nationaux et locaux. De plus, pourraient apparaître des effets pervers, dont la conversion des forêts naturelles en plantations ligneuses à croissance rapide, qui présentent un meilleur potentiel de stockage du carbone, soit plus de crédits de pollution mais au détriment de la biodiversité. En 1997, les incendies de forêt en Indonésie ont été la première opportunité de pointer la responsabilité des entreprises forestières et agro-industrielles, et ils ont permis de souligner que dans les pays défavorisés, c'est surtout la pauvreté des paysans, plutôt que la croissance démographique, qui constitue le principal facteur dégradant de l'environnement forestier et que les solutions passent probablement par des soutiens aux agricultures paysannes et à la promotion de systèmes de production durables, protégés par des mécanismes institutionnels d'éco-certification internationale, garantissant par ailleurs le maintien des savoirs traditionnels forestiers. De nos jours, la déforestation, c'est-à-dire le processus de régression du couvert forestier, est relativement médiatisée et perçue comme une catastrophe écologique à l'échelle planétaire. Bien que les chiffres relatifs à la déforestation soient l'objet de vives controverses, les institutions internationales estiment que 134 millions d'hectares de forêt ont été détruits entre 1980 et 1990 et 154 millions d'hectares au cours de la décennie 1990-200012. Le rythme s'est fortement accéléré, que ce soit en Amazonie ou en Indonésie et en Malaisie pour exploiter l'huile de palme qui envahit dorénavant l'alimentation industrielle occidentale. Au cours des derniers mois de 2007, selon l'Institut National Brésilien pour la Recherche spatiale, les premières analyses des images satellites laissent apparaitre une perte d'environ $3200 \mathrm{~km}^{2}$ et l'étude définitive des données pourrait faire ressortir une surface détruite deux fois supérieure. Cette évolution est directement liée à l'agro-productivisme intensif du soja transgénique principalement, et à la forte croissance de la demande en carburants d'origine végétale ${ }^{13}$ qui nécessitent l'exploitation ultra mécanisée de surfaces agricoles de plus en plus importantes, ce qui conduit au dépeuplement progressif des zones de culture. Les enjeux et les intérêts de la gestion forestière, tant privés que nationaux, conduisent à de vives controverses, que la constitution d'un Forum intergouvernemental sur les forêts en 1995 sous l'égide de la Commission du Développement Durable (CDD) des Nations Unies $^{14}$ n'a pas encore vraiment permis de résoudre.

${ }^{12}$ Soit un taux annuel de déforestation d'environ $4 \%$ à l'échelle mondiale pour la dernière décennie.

${ }^{13}$ La demande mondiale d'agro-carburant met très gravement en jeu le renouvellement des sols et la sécurité alimentaire (Ferrone, 2008)

14 Organe d'échange d'informations sur les politiques forestières nationales et lieu de débat sur les critères et les indicateurs de développement durable. 


\section{LA SÉCURITÉ ALIMENTAIRE ET LE PATRIMOINE DE BIODIVERSITÉ}

Bien que la communauté scientifique considère généralement que la Terre peut produire assez de nourriture pour tous, on estime à $20 \%$ environ la proportion de population mondiale souffrant d'alimentation déficiente, la pauvreté constituant le plus souvent le discriminant principal : on évalue à 1,3 milliard d'individus le nombre de ceux qui vivent avec moins de $1 \$$ par jour! De la production à la consommation des aliments, l'homme est intervenu à tous les niveaux de la chaîne alimentaire pour améliorer leurs qualités nutritionnelles, hygiéniques et organoleptiques. Ce faisant, les compagnies de biotechnologie contrôlent dorénavant largement l'offre alimentaire et disposent de multiples brevets à tous les stades de la chaine alimentaire, depuis les semences jusqu'aux aliments conditionnés. Mais le transfert de gènes d'une espèce à l'autre présente des risques imprévisibles, ce pourquoi les pollutions chimiques et génétiques seront probablement parmi les plus grands défis que devra affronter la population de la planète au cours du XXIème siècle. Les dérives de l'agroindustrie et des produits alimentaires post-modernes fondent le procès d'une technoscience qui détruit les représentations patrimoniales de la nature et constitue un danger pour la santé de la reproduction humaine. Le transgénisme et les biotechnologies modernes induisent de profondes mutations que les populations ignorent le plus souvent, les firmes alimentaires restant pour l'heure très libres de leurs processus de transformation : elles incorporent aux plantes, pour des raisons de rentabilité, des gènes qui comportent une résistance aux pesticides; les brevets déposés par les grandes firmes de l'agrochimie leur assurent ensuite une rente de situation difficilement réversible. La totalité du marché des semences transgéniques est détenue par les cinq premières sociétés de génie génétique (AstraZeneca, DuPont, Monsanto, Novartis et Aventis) et ce marché, évalué à plus de trois milliards de dollars, est largement dominé par la seule firme Monsanto. De l'agriculture à la chimie et à la pharmacie, en passant par les processus de décontamination de l'environnement ou la fabrique de matières plastiques, les biotechnologies font l'objet d'applications multiples et hautement rentables, mais en parallèle, de la vache folle au poulet à la dioxine, en passant par les céréales transgéniques, la viande aux hormones... ces mutations alimentaires traduisent bien les dysfonctionnements auxquels est confrontée la population de notre monde moderne. La liste des dérives de la production alimentaire a de quoi préoccuper les consommateurs qui voient disparaittre sous leurs yeux le monde paysan, au profit de l'industrie agroalimentaire qui assimile la biodiversité à une marchandise. Dans les systèmes économiques modernes, les agriculteurs, devenus "exploitants agricoles, subissent des évolutions technologiques et commerciales opaques, fruits des manipulations des firmes multinationales agroalimentaires. Sous couvert d'innovation, la qualité de l'alimentation se dégrade sensiblement et l'on mange toujours plus "d'objets comestibles non

Mondes en Développement Vol.36-2008/2-n¹42 
identifiés" que les consommateurs ne contrôlent plus. Or, les gouvernements commencent à prendre conscience que des protections sont difficiles à instaurer : il s'agit essentiellement d'embargos, le plus souvent curatifs, mis en place après qu'épidémies et bactéries aient traversé toutes les frontières ! Pourtant, la situation parait susceptible d'évoluer vers des stratégies préventives. De fait, le débat concernant les facultés de discernement des consommateurs au regard de l'offre alimentaire est loin d'être clos, d'autant que la demande de la grande majorité de la population mondiale répond aux seuls critères de prix à bon marché des produits alimentaires, sans autre considération. Dans ces conditions, il est clair que la conjugaison du cycle infernal de la pauvreté et des faibles niveaux d'instruction de la grande majorité de la population mondiale promet de beaux jours à l'alimentation industrielle et à la consommation de masse standardisée, quelles que soient les manipulations effectuées! Le contôle progressif de la semence par l'agro-industrie tend à supprimer le droit inaliénable de chaque producteur à ressemer sa récolte et constitue une grave préoccupation pour l'avenir, d'autant que la semence industrielle ne peut vivre sans pesticides, engrais chimiques ou manipulations génétiques et que les monocultures extensives réduisent toujours plus considérablement le renouvellement de la biodiversité.

\section{CONCLUSION}

Les États-nations ne peuvent plus prétendre contrôler seuls les gigantesques flux de capitaux et les marchés de consommation qui sous-tendent les dynamiques de croissance économique, d'emploi et de migrations ; de même, ils ne peuvent plus résoudre de manière autonome les problèmes d'environnement. Il est devenu nécessaire de mettre en œuvre des régulations environnementales encore très incertaines et faiblement institutionnalisées, que la recomposition des territoires et de leurs usages, l'urbanisation, la croissance des infrastructures et des activités polluantes... mettent chaque jour plus en danger. C'est toute une gestion durable des espaces, et parfois des espèces, qu'il convient de mettre en œuvre par delà des régulations démographiques relativement prévisibles. Le modèle occidental de développement ne peut être généralisé à tous les humains : il n'est pas durable ! La croissance exponentielle de la consommation de ressources et de la production de déchets est incompatible avec les capacités finies de régulation de la planète.

Une avancée intéressante résultant de la relative prise de conscience des problèmes démo-environnementaux est l'élaboration d'indicateurs ${ }^{15}$ pour disposer de mesures comparatives dans le temps et dans l'espace. De fait, les problèmes sont multiscalaires allant du niveau local au niveau global et tant les administrations que les entreprises, les medias ou l'opinion publique, les gouvernants et les organismes non-gouvernementaux, tous ont besoin de

${ }^{15}$ Cf. notamment le compendium de l’OCDE, données sur l'environnement, 2004, et mise à jour partielle 2007.

Mondes en Développement Vol. 36-2008/2-n¹42 
données fiables et harmonisées sur les relations population-environnementdéveloppement. L'OCDE a ainsi élaboré un compendium qui fait l'objet de mises à jour de plus en plus précises et tend à servir de référence, parmi d'autres.

Créé par le plan d'action adopté mi-décembre à la conférence de l'ONU à Bali, le Groupe spécial sur le "post 2012" s'est réuni pour la première fois, du 31 mars au 4 avril 2008, à Bangkok pour négocier les suites à donner aux engagements de réduction de gaz à effet de serre (GES), fixés par le protocole de Kyoto, qui expirent fin 2012. Les négociateurs devront adopter, entre autres, "un objectif global à long terme de réduction des émissions" et convenir des moyens de "renforcer l'action" (adaptation aux impacts du changement climatique ; mise au point et transfert Nord-Sud des technologies propres ; apport de ressources financières et d'investissements pour aider les populations du Sud).

Le monde connaittra ces cinquante prochaines années les plus importants contrastes démographiques de son histoire : douze pays verront leur population tripler ; cinquante et un verront chuter la leur, principalement en Europe. Les pays en transition démo-économique devront accueillir les 2,5 milliards d'hommes supplémentaires qui s'y concentreront dans les prochaines décennies, tout en tentant de pallier la dégradation des agro-systèmes et de s'adapter aux mutations environnementales et climatiques probables. Les dérives de l'industrialisation, de l'agriculture et du productivisme alimentaire instruisent progressivement le procès d'un développement économique toujours plus inégalitaire pour les hommes et dangereux pour la planète. La mise en évidence de ces perspectives préoccupantes, et des défis qu'elles suscitent, souligne l'urgence et l'ampleur des changements qui doivent être introduits dans les politiques publiques environnementales parce qu'ils sont absolument nécessaires à la viabilité de la planète.

\section{BIBLIOGRAPHIE}

AUBERTIN C., VIVIEN F.-D. (1998) Les enjeux de la biodiversité, Paris, Economica, Col. Poche Environnement.

CHETAILLE A., TAVERNIER K. (2003) Echec de la cinquième conférence ministérielle de l'OMC à Cancun : vers une crise du système commercial multilatéral ?, Solagral, (www.solagral.org).

CNUCED (2003) Rapport sur le commerce et le développement : Accumulation de capital, croissance économique et transformation structurelle, UNCTAD/TDR/2003, New York et Genève.

DOMENACH H. (2007) Démographie et environnement: vers une régulation planétaire ? La Jaune et la Rouge, École Polytechnique, $\mathrm{n}^{\circ}$ 627, 16-22

DOMENACH H., PICOUET M. (eds) (2004) Environnement et population : la durabilité en question, Collection Populations, Paris, L'Harmattan, 227 p.

DOMENACH H., PICOUET M. (2000) Population et environnement, Paris, PUF, collection "Que sais-je?" n 3556. 
EL-HINNAWI E. (1985) Environmental Refugees, Nairobi, United Nations Environmental Program.

FERRONE G. (2008) 2030 le kerach écologique, Paris, Grasset, 286 p.

GIEC (2007) 4ème Rapport d'évaluation du Groupe d'experts intergouvernemental sur l'évolution du climat, Bruxelles.

HAMELIN P. (2004) La transformation des espaces ruraux ; de la production agricole au développement rural, in Domenach H., Picouet M. (eds), 77-100.

MEADOWS Do., MEADOWS De., RANDERS J. (2004) Limits to Growth, Paperback format, Chelsea Green Pub Co.

PINSON D. (2004), Environnement et urbanisation, in Domenach H., Picouet M. (eds), 33-51.

OECD, (2004), Environmental data : Compendium, 313 p.

QUENAULT B. (2001) Conférence de Rio, développement durable, organisation mondiale de l'environnement, in Lorot P. (dir.) Dictionnaire de la mondialisation, Paris, Ellipses.

SEN A. (2000) Un nouveau modèle économique. Développement, justice, liberté, Paris, O. Jacob.

UN report of the Conference on Environment and Development, Rio de Janeiro (1992) Vol.1, Resolutions adopted, E.93.I.8.

UN report of the International Conference on Population and Development (1994) Vol.1, Resolutions adopted, E.95.XIII.18, Le Caire.

UNITED NATIONS (2007a) World Urbanization Prospects: The 2006 Revision, Division de la population, New York.

UNITED NATIONS (2007b), World Population Prospects: The 2006 Revision Highlights, ST/ESA/SER.A./261/ES, New York.

UNITED NATIONS (2006) World Urbanization Prospects: The 2005 Revision, Division de la population, Département des affaires économiques et sociales, New York.

UNITED NATIONS DEVELOPMENT PROGRAMME (2004) Reducing disaster risk A challenge for development (Global Report), Bureau for Crisis Prevention and Recovery, http://www.undp.org/bcpr, 146 pp. 
Les grandes tendances démographiques et l'environnement : l'enjeu d'une planète viable Hervé DOMENACH

Cet article traite des projections démographiques et des mutations environnementales, en présentant une vision des défis qui en résultent pour les institutions. Sont examinés, à travers le prisme de la durabilité de nos modes de développement, le processus de vieillissement, la mobilité des hommes et les mutations démo-spatiales, le renouvellement des ressources aquatiques et forestières, la sécurité alimentaire et le patrimoine de biodiversité...

Mots-clés : Démographie, environnement, développement, analyse démospatiale, mobilité, ressources naturelles, biodiversité, eau, forêt.

Classification JEL : Q56, O15, P28

\section{Main demographic and environmental tendencies: the stake of a viable planet Hervé DOMENACH}

This article treats demographic projections and environmental changes, by presenting a vision of the challenges which result from it for the institutions. Are examined, through the prism of the durability of our modes of development, the process of ageing, the mobility of the men and the spatial demographic changes, the renewal of the watery and forest resources, the food safety and the inheritance of biodiversity...

Keywords: Demography, environment, development, analyze demonstrationspace, mobility, natural resources, biodiversity, water, forest.

\section{Classification JEL : Q56, O15, P28}

